\title{
Screening for genes, transcription factors and miRNAs associated with the myogenic and osteogenic differentiation of human adipose tissue-derived stem cells
}

\author{
LIANGLIANG QUAN, YANG WANG, JIULONG LIANG, TAO QIU, HONGYI WANG, \\ YE ZHANG, YU ZHANG, QIANG HUI and KAI TAO \\ Department of Plastic Surgery, General Hospital of Shenyang Military Area \\ Command, PLA, Shenyang, Liaoning 110016, P.R. China
}

Received July 9, 2015; Accepted September 9, 2016

DOI: $10.3892 / \mathrm{ijmm} .2016 .2788$

\begin{abstract}
In the present study, we aimed to reveal the molecular mechanisms responsible for the differentiation of human adipose tissue-derived stem cells (hASCs) into myocytes and osteoblasts. Microarray data GSE37329 were obtained from the Gene Expression Omnibus database, including three hASC cell lines from healthy donors, two osteogenic lineages and two myogenic lineages from the in vitro-induction of hASCs. Differentially expressed genes (DEGs) in the two lineages were firstly screened. Subsequently, the underlying functions of the two sets of DEGs were investigated by Gene Ontology function and Kyoto Encyclopedia of Genes and Genomes pathway enrichment analysis, followed by protein-protein interaction (PPI) network construction. Regulatory relationships between transcription factors (TFs) and microRNAs (miRNAs or miRs) with target genes were finally explored using different algorithms. A total of 665 and 485 DEGs were identified from the hASC-derived myogenic and osteogenic lineages, respectively. The shared upregulated genes $(n=205)$ in the two sets of DEGs were mainly involved in metabolism-related pathways, whereas the shared downregulated genes $(n=128)$ were significantly enriched in the transforming growth factor- $\beta$ (TGF- $\beta$ ) signaling pathway. Four genes, vascular endothelial growth factor A (VEGFA), fibroblast growth factor 2 ( $F G F 2$ ), nerve growth factor $(N G F)$ and interleukin $1 \mathrm{~B}(I L I B)$, presented with relatively higher degrees in both PPI networks. The transcription factor RAD21 was predicted to target shared upregulated and downregulated genes as well as specific downregulated genes in the myogenic and the osteogenic lineages. In addition, miRNA-DEG interaction analysis revealed that hsa-miR-1 regulated the most shared DEGs in the two lineages. There may
\end{abstract}

Correspondence to: Dr Kai Tao, Department of Plastic Surgery, General Hospital of Shenyang Military Area Command, PLA, 83 Wenhua Road, Shenhe, Shenyang, Liaoning 110016, P.R. China E-mail:kaitaoktk@163.com

Key words: human adipose tissue-derived stem cells, differentiation, myocyte, osteoblast, transcription factor, microRNA be a correlation between the four genes, VEGFA, FGF2, ILIB and $N G F$, and the differentiation of hASCs into myocytes and osteoblasts. The TF RAD21 and hsa-miR-1 may play important roles in regulating the expression of differentiation-associated genes.

\section{Introduction}

Human adipose tissue-derived stem cells (hASCs) are an attractive cell type for tissue engineering which may be harvested by direct excision or liposuction from human adipose tissue. Physiologically, hASCs are capable of differentiating into various lineages, such as adipocytes, osteoblasts, myocytes and chondrocytes $(1,2)$. The ability of hASCs to undergo multilineage differentiation has attracted increasing interest in their use clinically and in regenerative medicine (3). A number of studies have suggested that hASCs possess significant potential for tissue rescue in multiple animal models, including heart failure, myocardial infarction, bone formation and wound healing, by differentiating into a variety of lineages (4-6).

Many factors have been reported to be involved in the mechanisms of hASC differentiation. Nutritional and hormonal signaling affects hASC differentiation in a negative or a positive manner, and the molecules involved in cell-matrix or cell-cell interactions play key roles in regulating the differentiation process (7-9). It is well known that fibroblast growth factor $2(F G F 2)$ inhibits the osteogenic differentiation of hASCs whereas it promotes chondrogenesis $(10,11)$. Moreover, microRNA (miRNA or miR)-26a has been shown to modulate the late stage of osteoblast differentiation by targeting the transcription factor (TF) SMAD family member 1 (SMAD1) (4). The upregulation of miRNA-22 has been proved to promote the osteogenic differentiation of human adipose tissue-derived mesenchymal stem cells by suppressing histone deacetylase 6 (HDAC6) expression (12). Furthermore, hASCs are capable of differentiating into skeletal myocytes and cardiomyocytes under specific conditions (incubation in myogenic medium) $(13,14)$. In vitro, sphingosylphosphorylcholine and transforming growth factor- $\beta$ (TGF- $\beta$ ) induced the expression of smooth muscle-associated markers including $\alpha$-smooth muscle actin, calponin and SM22 in hASCs $(15,16)$. Numerous 
studies have been performed to reveal the molecular mechanisms controlling the differentiation of hASCs (7-16). However, the mechanisms responsible for the regulation of myocyte and osteocyte differentiation remain largely unknown.

Increasing evidence has proved that the conversion of hASCs into differentiated myocytes and osteocytes involves changes in gene expression which are mainly regulated by miRNAs and TFs $(17,18)$. For instance, Luzi et al (4) showed that miR-26a expression was increased during hASC differentiation, whereas the expression of SMAD1 was complementary to that of miR-26a. In addition, Kim et al (17) reported that miR-196a regulates the differentiation and proliferation of hASCs by modulating the levels of the HOXC 8 transcription factor.

To gain further insight into the molecular mechanisms responsible for the differentiation of hASCs into myocytes and osteocytes, we re-analyzed the microarray data GSE37329 through the identification of differentially expressed genes (DEGs) in hASC-derived myocytes and osteocytes compared with hASCs, as well as through functional annotation and protein-protein interaction (PPI) network construction. Furthermore, TFs and miRNAs targeting the DEGs were predicted and functionally analyzed.

\section{Materials and methods}

Gene datasets. The gene expression profile of GSE37329 was retrieved from the Gene Expression Omnibus (GEO) database available at http://www.ncbi.nlm.nih.gov/geo/ (19). This dataset was deposited by Berdasco et al (19) on October 3, 2013 and was based on GPL11532 platform (Affymetrix Human Gene 1.1 ST array, Santa Clara, CA, USA). A total of 7 samples were available for further study, including three hASC cell lines from healthy donors, two osteogenic lineages and two myogenic lineages which were all obtained through the in vitro induction of hASCs.

Data preprocessing. The raw expression data (Affymetrix CEL files) were firstly preprocessed by the Robust Multiarray Average (RMA) normalization approach of Bioconductor affy package in R (20) (http://www.bioconductor.org), which returned the expression signals of each probe as $\log 2$ scale. When different probes were mapped to the same gene, the mean value of the probes was considered as the gene value. Subsequently, the probe serial numbers in the matrix were transformed into gene names using the platform R/Bioconductor note package of the dataset chip. The matrix consisting of 20,253 genes was finally acquired.

Screening of DEGs. To screen out the DEGs in the in vitro-obtained osteogenic and myogenic lineages derived from hASCs compared with the freshly isolated hASCs obtained from healthy donors, respectively, Linear Models for Microarray Data (Limma) package of Bioconductor (21) was applied in the comparisons (osteogenic lineages vs. hASCs and myogenic lineages vs. hASCs). Unadjusted P-values were calculated using the Student's t-test. Genes with $\mathrm{P}<0.05$ and $\log 2 \mathrm{FC}$ (fold change) $\mid \geq 1$ were considered to be differentially expressed. Hierarchical cluster analysis with the eligible DEGs was then performed in order to identify clusters of samples and genes.
Functional annotation of the DEGs. Functional enrichment of the two sets of DEGs in the osteogenic and the myogenic lineages in vitro-induced from the hASCs was assessed based on the biological process (BP) category in Gene Ontology (GO) (22) and Kyoto Encyclopedia of Genes and Genomes (KEGG) annotation terms (23). GO and KEGG signaling pathway analyses were performed using the GO Function package (version 1.14.0) in Bioconductor (http://www.bioconductor.org/packages/ release/bioc/html/GOFunction.html) (24), which conducted the standard hypergeometric test. A P-value $<0.05$ was considered to indicate a statistically significant difference.

PPI network construction. Search Tool for the Retrieval of Interacting Genes (STRING; http://string-db.org/) is an online database which is comprised of more than 1,100 completely sequenced organisms and includes experimental as well as predicted interaction information (25). The up- and downregulated genes in both sets of DEGs verified above were directly mapped to the STRING database in order to acquire significant PPI pairs which were previously verified by experiments, text mining and/or co-expressed analysis, respectively. Notable PPI pairs in which both of the genes were differentially expressed and the medium confidence was $\geq 0.4$ were integrated to construct a PPI network. The network was visualized using CytoScape (26), available at http://www.cytoscape.org. Considering the complexity of PPI networks, we computed the degree of each node by measuring the numbers of links of the node in the network.

Computational identification of TFs. To determine the common mechanism responsible for the differentiation of hASCs into myocytes and osteocytes, DEGs shared in the osteogenic and the myogenic lineages were screened out. KEGG pathway enrichment analysis of the shared up- and downregulated genes was performed, respectively. P-values were calculated using hypergeometric distribution and a $\mathrm{P}$-value $<0.05$ was considered to indicate a significant pathway.

To further explore the molecular mechanism, eukaryotic TFs for the shared and unshared DEGs in osteogenic and myogenic lineages were collected based on the the Encyclopedia of DNA Elements (ENCODE) data from the USCS Genome Browser (27) available at http://genome. ucsc.edu/. P-values were calculated using Fisher's exact test and adjusted using the Benjamini and Hochberg method to define the false discovery rate (FDR). Only the results with an FDR $<5.5$ E-06 were considered to be significant.

miRNAs-target gene interaction network construction. To better understand the function of miRNAs in regulating the differentiation of hASCs, miRNAs targeting the shared up- and downregulated DEGs screened above were predicted using the miRecords database (28) available at http://c1.accurascience. com/miRecords/ and the miRWalk database (29) available at http://zmf.umm.uni-heidelberg.de/apps/zmf/mirwalk2/. The miRNA-target interactions that were presented in miRecords and/or miRWalk and verified by experiment were used for the construction of the miRNA-mRNA interaction network. The network was visualized using CytoScape and the degree of each miRNA node was also measured. Furthermore, the predicted miRNAs were annotated with BP terms in the GO database. 
A

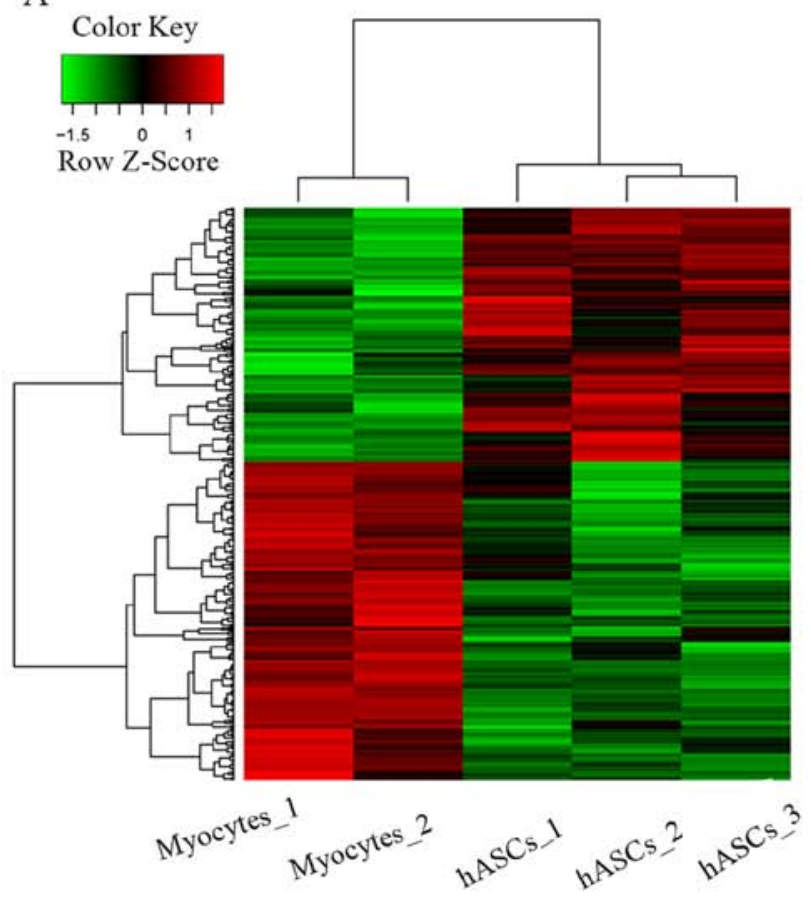

B

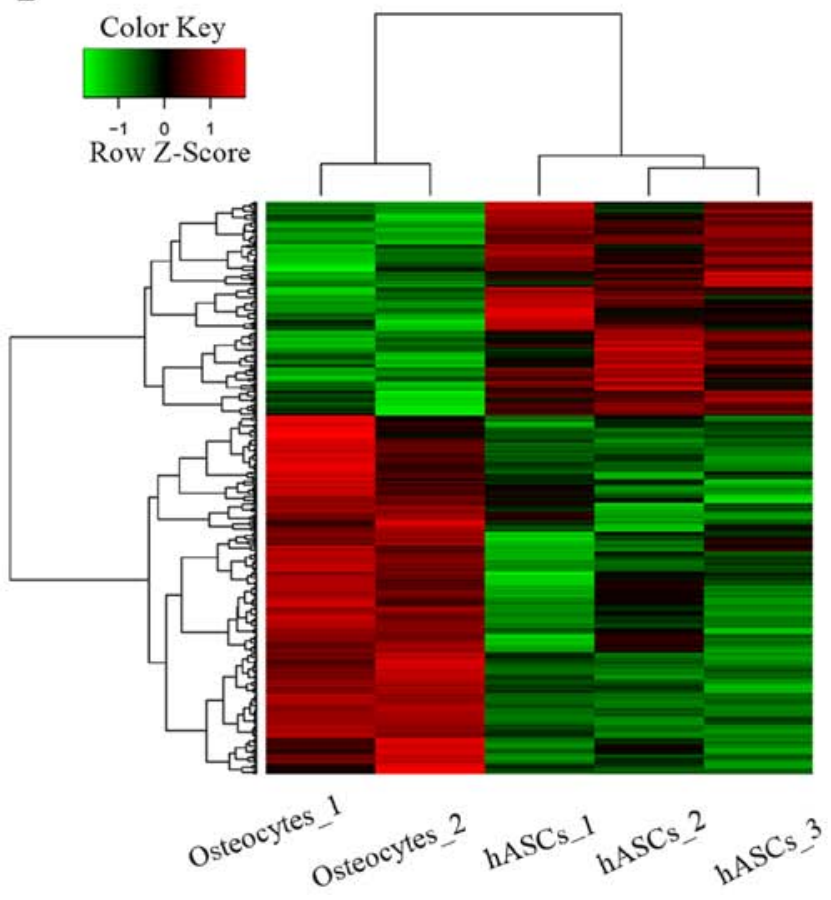

Figure 1. Hierarchical clustering of significantly differentially expressed genes in myocytes and osteocytes differentiated from human adipose tissue-derived stem cells (hASCs). (A) Clustering of genes in myocytes. (B) Clustering of genes in osteocytes. Red represents high expression, and the deeper the red color, a higher expression value. Green represents low expression, and a deeper green color, a lower the expression value.

P-values were calculated using hypergeometric distribution and GO terms with a P-value $<0.05$ were defined as significantly enriched.

\section{Results}

Screening of DEGs. Compared with the hASCs, 665 DEGs in myogenic lineages (370 up- and 295 downregulated genes) and 485 DEGs in osteogenic lineages (304 up- and 181 downregulated genes) were finally identified. The two sets of eligible DEGs were evaluated using unsupervised hierarchical clustering. As shown in Fig. 1, DEGs were found in different samples.

Annotating the biological functions of DEGs. To elucidate the functions of DEGs, the up- and downregulated genes in the in vitro-obtained myogenic and osteogenic lineages were mapped to BP terms in the GO database, and the top $10 \mathrm{GO}$ terms are shown in Tables I and II, respectively. Briefly, the upregulated genes identified from the myogenic lineages were mainly involved in the regulation of multicellular organismal processes, inflammatory responses and cellular responses to chemical stimuli, whereas the downregulated genes were mainly involved in the regulation of multicellular organismal processes, single-multicellular organism processes, single-organism developmental processes and multicellular organismal development. On the other hand, the upregulated genes in the osteogenic lineages were mainly associated with responses to stimuli, regulation of multicellular organismal processes and regulation of localization, whereas the downregulated genes were mainly associated with anatomical structure development, system development and tissue development.
KEGG pathway enrichment analysis was used to further understand the biological functions of the DEGs. Analysis of the myogenic lineages revealed that the upregulated genes mainly participated in neuroactive ligand-receptor interactions and drug metabolism-cytochrome P450 pathways (Table III), which was the same as the upregulated genes in the osteogenic lineages (Table IV). By contrast, the downregulated genes in the myogenic lineages were mainly enriched in pathways in cancer, ECM-receptor interactions and focal adhesion (Table III), while the downregulated genes in the osteogenic lineages were mainly involved in the TGF- $\beta$ signaling pathway and pathways in cancer (Table IV).

PPInetwork construction. There were 363 nodes and 996 edges in the PPI network of DEGs in myogenic lineages (Fig. 2). Based on the number of links, the top 8 nodes were identified as vascular endothelial growth factor A (VEGFA; degree, 57), interleukin (IL)6 (degree, 49), FBJ murine osteosarcoma viral oncogene homolog (FOS; degree, 41), FGF2 (degree, 37), jun proto-oncogene (JUN; degree, 35), IL1B (degree, 34), phosphoinositide-3-kinase, regulatory subunit 1 (PIK3R1; degree, 28) and nerve growth factor (NGF; degree, 27). In addition, 246 nodes and 520 edges constructed the PPI network of DEGs in the osteogenic lineages (Fig. 3), and the top 8 nodes were VEGFA (degree, 40), endothelin 1 (EDN1; degree, 24), IL1B (degree, 24), FGF2 (degree, 22), insulin-like growth factor 1 (IGF1; degree, 21), leptin (LEP; degree, 19), NGF (degree, 18) and matrix Gla protein (MGP; degree, 14). Considering the higher degree of VEGFA, IL1B, FGF2 and NGF in both networks, we hypothesized that these four genes play similar roles in the differentiation of hASCs into the two cell types. 
Table I. Top 10 enriched GO terms in the BP category for both upregulated and downregulated differentially expressed genes in myocytes.

\begin{tabular}{|c|c|c|c|c|c|}
\hline & & GO ID & Name of BP & Count & P-value \\
\hline \multirow[t]{10}{*}{ Up } & $\mathrm{BP}$ & GO:0051239 & Regulation of multicellular organismal process & 86 & $2.28 \mathrm{E}-11$ \\
\hline & & GO:0006954 & Inflammatory response & 35 & 2.20E-09 \\
\hline & & GO:0070887 & Cellular response to chemical stimulus & 83 & 2.25E-09 \\
\hline & & GO:0042221 & Response to chemical & 115 & 2.87E-08 \\
\hline & & GO:0050896 & Response to stimulus & 198 & $6.91 \mathrm{E}-08$ \\
\hline & & GO:0032879 & Regulation of localization & 70 & 7.58E-08 \\
\hline & & GO:0050727 & Regulation of inflammatory response & 20 & 7.83E-08 \\
\hline & & GO:0006805 & Xenobiotic metabolic process & 16 & 7.94E-08 \\
\hline & & GO:0050793 & Regulation of developmental process & 67 & 8.64E-08 \\
\hline & & GO:0071466 & Cellular response to xenobiotic stimulus & 16 & 8.68E-08 \\
\hline \multirow[t]{10}{*}{ Down } & $\mathrm{BP}$ & GO:0001944 & Vasculature development & 43 & 0 \\
\hline & & GO:0007275 & Multicellular organismal development & 147 & 0 \\
\hline & & GO:0009653 & Anatomical structure morphogenesis & 104 & 0 \\
\hline & & GO:0009888 & Tissue development & 75 & 0 \\
\hline & & GO:0030154 & Cell differentiation & 118 & 0 \\
\hline & & GO:0032501 & Multicellular organismal process & 173 & 0 \\
\hline & & GO:0032502 & Developmental process & 156 & 0 \\
\hline & & GO:0044707 & Single-multicellular organism process & 172 & 0 \\
\hline & & GO:0044767 & Single-organism developmental process & 153 & 0 \\
\hline & & GO:0048731 & System development & 139 & 0 \\
\hline
\end{tabular}

Up, upregulated; down, downregulated; GO, gene ontology; BP, biological process.

Table II. Top 10 enriched GO terms in the BP category for both upregulated and downregulated differentially expressed genes in osteocytes.

\begin{tabular}{|c|c|c|c|c|c|}
\hline & & GO ID & Name of BP & Count & P-value \\
\hline \multirow[t]{10}{*}{ Up } & \multirow[t]{10}{*}{$\mathrm{BP}$} & GO:0050896 & Response to stimulus & 174 & $2.59 \mathrm{E}-10$ \\
\hline & & GO:0006805 & Xenobiotic metabolic process & 15 & 3.63E-08 \\
\hline & & GO:0071466 & Cellular response to xenobiotic stimulus & 15 & $3.96 \mathrm{E}-08$ \\
\hline & & GO:0032879 & Regulation of localization & 61 & $5.48 \mathrm{E}-08$ \\
\hline & & GO:0009410 & Response to xenobiotic stimulus & 15 & $6.02 \mathrm{E}-08$ \\
\hline & & GO:0051239 & Regulation of multicellular organismal process & 64 & 4.22E-07 \\
\hline & & GO:0051049 & Regulation of transport & 48 & 4.74E-07 \\
\hline & & GO:0006954 & Inflammatory response & 27 & $5.39 \mathrm{E}-07$ \\
\hline & & GO:0051046 & Regulation of secretion & 26 & $7.18 \mathrm{E}-07$ \\
\hline & & GO: 1901700 & Response to oxygen-containing compound & 42 & $1.88 \mathrm{E}-06$ \\
\hline \multirow[t]{10}{*}{ Down } & \multirow[t]{10}{*}{$\mathrm{BP}$} & GO:0072358 & Cardiovascular system development & 33 & $4.90 \mathrm{E}-12$ \\
\hline & & GO:0072359 & Circulatory system development & 33 & $4.90 \mathrm{E}-12$ \\
\hline & & GO:0014706 & Striated muscle tissue development & 20 & $3.27 \mathrm{E}-11$ \\
\hline & & GO:0060537 & Muscle tissue development & 20 & $6.71 \mathrm{E}-11$ \\
\hline & & GO:0048731 & System development & 74 & $1.71 \mathrm{E}-10$ \\
\hline & & GO:0001944 & Vasculature development & 24 & $9.03 \mathrm{E}-10$ \\
\hline & & GO:0048856 & Anatomical structure development & 80 & $1.07 \mathrm{E}-09$ \\
\hline & & GO:0009888 & Tissue development & 42 & $3.06 \mathrm{E}-09$ \\
\hline & & GO:2000026 & Regulation of multicellular organismal development & 37 & $3.14 \mathrm{E}-09$ \\
\hline & & GO:0009653 & Anatomical structure morphogenesis & 51 & 4.97E-09 \\
\hline
\end{tabular}

Up, upregulated; down, downregulated; GO, gene ontology; BP, biological process. 
Table III. Top 10 enriched KEGG pathways of upregulated and downregulated differentially expressed genes in myocytes.

\begin{tabular}{|c|c|c|c|c|}
\hline & KEGG ID & Name & Count & P-value \\
\hline \multirow[t]{10}{*}{ Up } & 00982 & Drug metabolism - cytochrome P450 & 9 & $2.36 \mathrm{E}-05$ \\
\hline & 00350 & Tyrosine metabolism & 5 & 0.001759753 \\
\hline & 05145 & Toxoplasmosis & 8 & 0.007544112 \\
\hline & 00460 & Cyanoamino acid metabolism & 2 & 0.009086159 \\
\hline & 00071 & Fatty acid metabolism & 4 & 0.013452544 \\
\hline & 05014 & Amyotrophic lateral sclerosis (ALS) & 4 & 0.027079145 \\
\hline & 04080 & Neuroactive ligand-receptor interaction & 11 & 0.032800823 \\
\hline & 00603 & Glycosphingolipid biosynthesis - globo series & 2 & 0.035670336 \\
\hline & 00590 & Arachidonic acid metabolism & 4 & 0.038155628 \\
\hline & 00120 & Primary bile acid biosynthesis & 2 & 0.045739216 \\
\hline \multirow[t]{10}{*}{ Down } & 04512 & ECM-receptor interaction & 12 & $5.83 \mathrm{E}-08$ \\
\hline & 04350 & TGF- $\beta$ signaling pathway & 11 & $4.72 \mathrm{E}-07$ \\
\hline & 05323 & Rheumatoid arthritis & 10 & $8.16 \mathrm{E}-06$ \\
\hline & 04510 & Focal adhesion & 14 & $2.53 \mathrm{E}-05$ \\
\hline & 04640 & Hematopoietic cell lineage & 9 & 4.23E-05 \\
\hline & 05200 & Pathways in cancer & 18 & $4.23 \mathrm{E}-05$ \\
\hline & 04514 & Cell adhesion molecules (CAMs) & 10 & 0.000217686 \\
\hline & 05144 & Malaria & 6 & 0.000396575 \\
\hline & 05412 & Arrhythmogenic right ventricular cardiomyopathy (ARVC) & 7 & 0.000505663 \\
\hline & 05217 & Basal cell carcinoma & 6 & 0.000599721 \\
\hline
\end{tabular}

ECM, extracellular matrix; TGF- $\beta$, transforming growth factor- $\beta$; up, upregulated; down, downregulated; KEGG, Kyoto Encyclopedia of Genes and Genomes.

Table IV. Top 10 enriched KEGG pathways of upregulated and downregulated differentially expressed genes in osteocytes.

\begin{tabular}{|c|c|c|c|c|}
\hline & KEGG ID & Name & Count & P-value \\
\hline \multirow[t]{10}{*}{ Up } & 00982 & Drug metabolism - cytochrome P450 & 10 & $5.30 \mathrm{E}-07$ \\
\hline & 00350 & Tyrosine metabolism & 6 & $7.68 \mathrm{E}-05$ \\
\hline & 04080 & Neuroactive ligand-receptor interaction & 14 & 0.000309764 \\
\hline & 04270 & Vascular smooth muscle contraction & 8 & 0.001033472 \\
\hline & 00460 & Cyanoamino acid metabolism & 2 & 0.006279036 \\
\hline & 00071 & Fatty acid metabolism & 4 & 0.006987698 \\
\hline & 00260 & Glycine, serine and threonine metabolism & 3 & 0.018947162 \\
\hline & 00590 & Arachidonic acid metabolism & 4 & 0.020746069 \\
\hline & 00603 & Glycosphingolipid biosynthesis - globo series & 2 & 0.025079512 \\
\hline & 00010 & Glycolysis/gluconeogenesis & 4 & 0.028465778 \\
\hline \multirow[t]{10}{*}{ Down } & 04350 & TGF- $\beta$ signaling pathway & 7 & $2.00 \mathrm{E}-05$ \\
\hline & 04610 & Complement and coagulation cascades & 4 & 0.005201809 \\
\hline & 05217 & Basal cell carcinoma & 3 & 0.018290654 \\
\hline & 04916 & Melanogenesis & 4 & 0.01930188 \\
\hline & 04972 & Pancreatic secretion & 4 & 0.01930188 \\
\hline & 04710 & Circadian rhythm - mammal & 2 & 0.020821702 \\
\hline & 00512 & Mucin type O-Glycan biosynthesis & 2 & 0.037222845 \\
\hline & 04360 & Axon guidance & 4 & 0.042211972 \\
\hline & 05200 & Pathways in cancer & 7 & 0.04712619 \\
\hline & 05020 & Prion diseases & 2 & 0.049293741 \\
\hline
\end{tabular}

TGF- $\beta$, transforming growth factor- $\beta$; up, upregulated; down, downregulated; KEGG, Kyoto Encyclopedia of Genes and Genomes. 


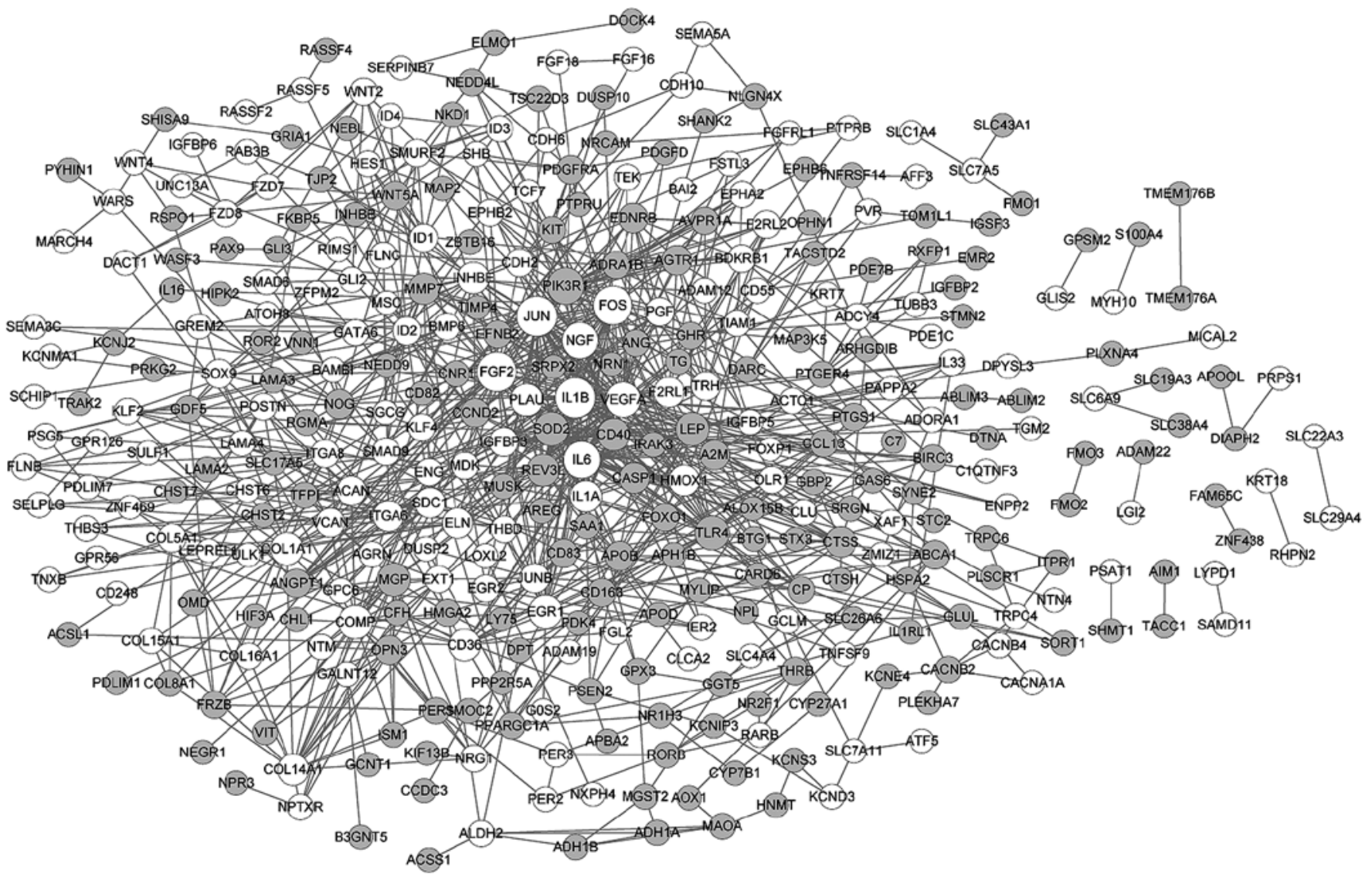

Figure 2. Protein-protein interaction network of differentially expressed genes in myocytes. Gray nodes represent downregulated genes and white nodes represent upregulated genes in myocytes. The higher the degree, the larger the node size.

CLDN11-JP2 PRDM16 SKIL, SAP30 ZNF395

CDH6 SEMA5A SRGN SRPX2 MSC SMURF2 PRKCH CY 27TA1

IGFBP2 CNR1 COH10 IGFBP3 SEMA3C HES ID3 SLTRK1 STOOA4 ID2) RASSF3

CD40 RGMA ADAM12 $A R H G D I B$ CD82 ETPBB1 GAS6 BNIP3 RASSF2 FLVCR2

RAB3C PRUNE2 NRN1 SHB A2M POGED INHBE STC1 8565 TSC22D 3 IP3 ZFPM2 TSPAN8 ENPP2 PBN2

GOSR UNC1BA PYCARD TEK GAN MLAU AR ZBTB16 SOX9 GREM2 GPR126 LGR5 GENT1 GALNT12

XAF1 RAB3D RIMS1 1 SLC4AA1 C1QTNF3 CP IGF1 FGF2 SDC1 FRBP5 NOY RSPO1 GATA6 GDF5 GALNT13 CLCA2

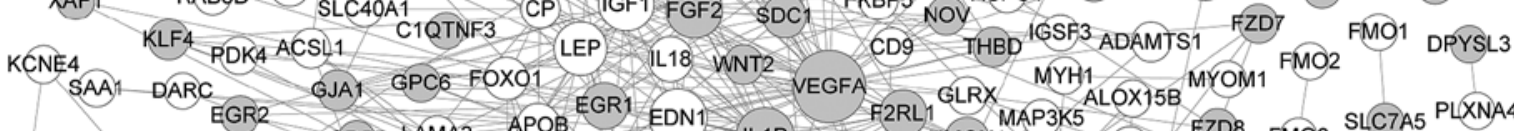

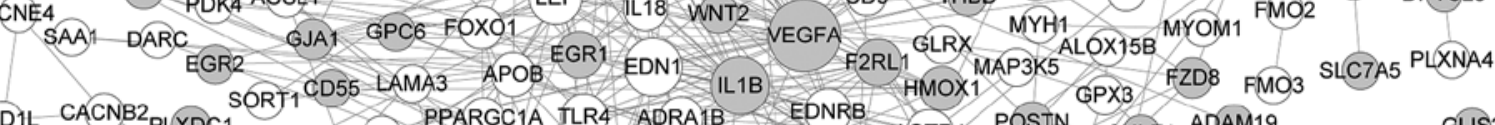

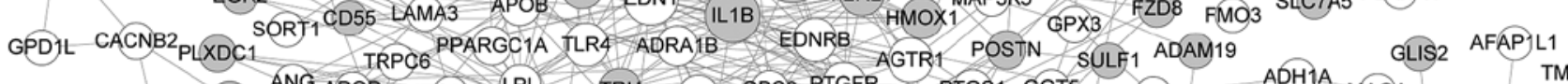
HSPA2 2 TRPC4 ANG APOD ITGA10 LPL TRH AVPRIAGPC3 PTGFR PGGS1 GGTS MGST2 ADH1A MAOA TMEMI76A

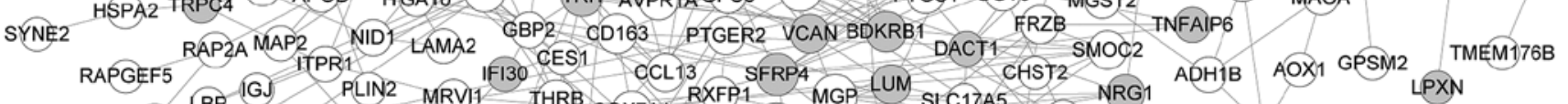

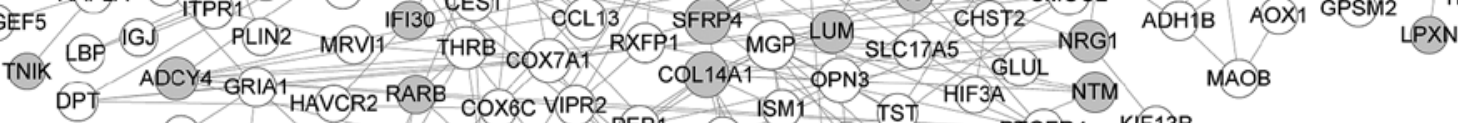

DPI SHISA9 HAVCR2 RARB COX6C VIPR2 PER1 SPARCL1 ISMT IST HIF3A NTGER4 KIF13B

GRIA3 CNII3 NR2FI NR1D2 PER3 CCDC3 COL1GA1 PII PROS1 SQRDL

CNKSR2 SAMD12ELTD1 PER2 APP1B1 COL8A1 NEGR1 FNDE1

PPP1R14A SLCBAA SLCGA9 RGS5 COL8A1 GNB

SLC19A3 SLC29A1 KONMA1 LIPG

Figure 3. Protein-protein interaction network of differentially expressed genes in osteocytes. Gray nodes represent downregulated genes and white nodes represent upregulated genes in osteocytes. The higher the degree, the larger the node size. 
Table V. Enriched KEGG pathways of shared genes between two groups (myocytes vs. hASCs and osteocytes vs. hASCs).

\begin{tabular}{|c|c|c|c|c|}
\hline & KEGG ID & Name of pathway & Count & P-value \\
\hline \multirow[t]{5}{*}{ Shared up } & 00982 & Drug metabolism - cytochrome P450 & 9 & $2.80 \mathrm{E}-07$ \\
\hline & 00350 & Tyrosine metabolism & 5 & 0.000155935 \\
\hline & 04080 & Neuroactive ligand-receptor interaction & 11 & 0.000601223 \\
\hline & 00071 & Fatty acid metabolism & 4 & 0.002082258 \\
\hline & 00460 & Cyanoamino acid metabolism & 2 & 0.003246415 \\
\hline \multirow[t]{4}{*}{ Shared down } & 04350 & TGF- $\beta$ signaling pathway & 7 & $3.78 \mathrm{E}-06$ \\
\hline & 04610 & Complement and coagulation cascades & 4 & 0.002133141 \\
\hline & 04916 & Melanogenesis & 4 & 0.008361484 \\
\hline & 04972 & Pancreatic secretion & 4 & 0.008361484 \\
\hline
\end{tabular}

TGF- $\beta$, transforming growth factor- $\beta$; up, upregulated; down, downregulated; KEGG, Kyoto Encyclopedia of Genes and Genomes; hASCs, human adipose-derived stem cells.

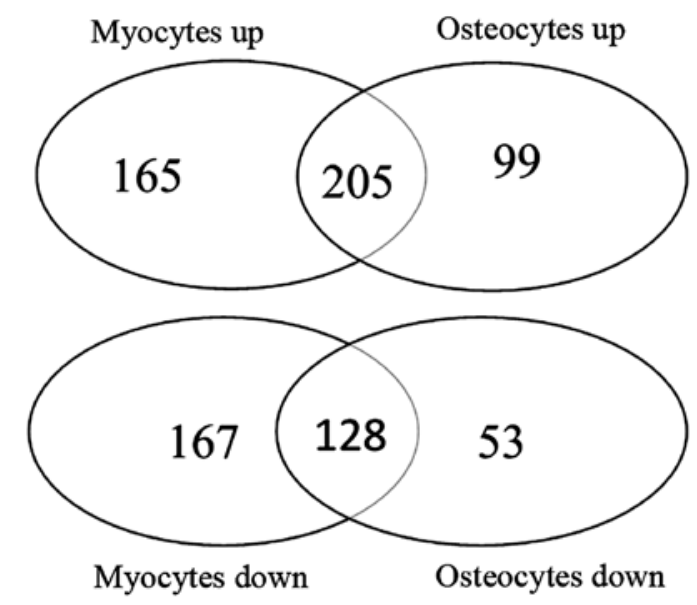

Figure 4. Venn diagram of shared genes and unshared genes identified by comparisons (myocytes vs. human adipose tissue-derived stem cells (hASCs) and osteocytes vs. hASCs). The overlapping genes are shared genes. The number of shared upregulated (up) and shared downregulated (down) genes is shown.

Enrichment analysis of TFs. To further explore the molecular mechanisms responsible for the differentiation of hASCs into myocytes and osteocytes, the shared and unshared DEGs in the in vitro-obtained osteogenic and myogenic lineages were analyzed, respectively (Fig. 4). The results of the KEGG enrichment analysis revealed that 205 shared upregulated genes were mainly involved in metabolism-related pathways, including drug metabolism and tyrosine metabolism, and 128 shared downregulated genes were significantly enriched in the TGF- $\beta$ signaling pathway (Fig. 4 and Table V).

The relationship between TFs and DEGs may aid in defining regulatory controls. Finally, a total of 27 TFs targeting the shared upregulated genes were predicted. In addition, $11 \mathrm{TFs}$, which are all involved in the targeting of the shared upregulated genes, were predicted to target the shared downregulated genes, including RAD21, zinc finger protein 263 (ZNF263), signal transducer and activator of transcription 3 (STAT3), RE1-silencing transcription factor (REST, also known as NRSF), tripartite motif containing 28 (TRIM28, also known as KAP1), GATA binding protein 2 (GATA2), CCCTC-binding

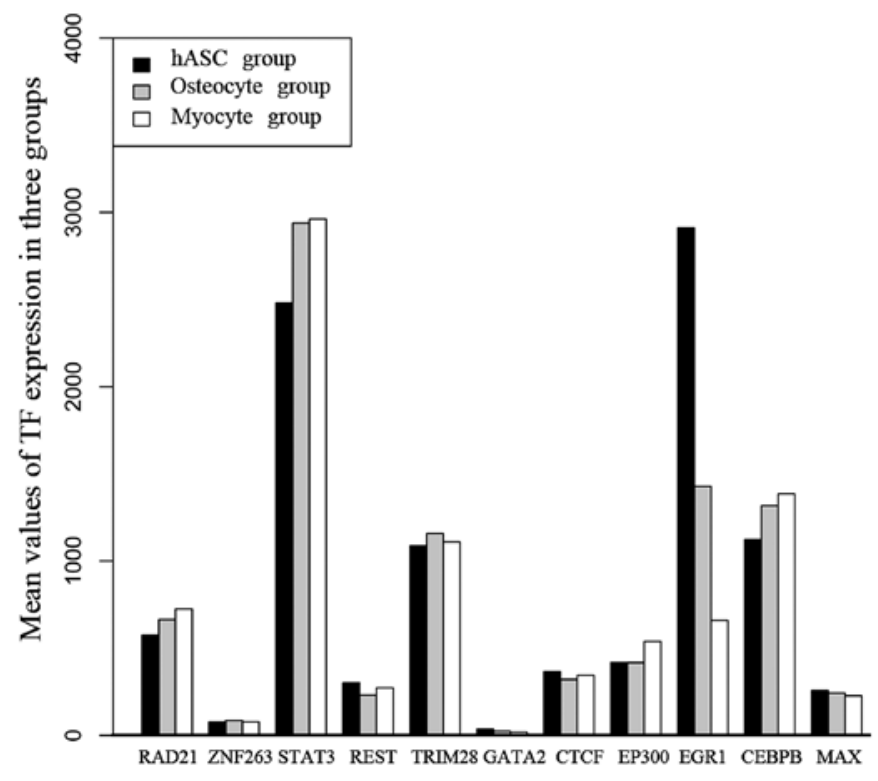

Figure 5. The mean expression of 11 transcription factors (TFs) in three cell lineages including human adipose tissue-derived stem cells (hASCs), myocytes and osteocytes. The horizontal axis represents TFs and the vertical axis represents the mean expression value of the TFs.

factor (CTCF), E1A binding protein p300 (EP300), early growth response 1 (EGR1), CCAAT/enhancer binding protein $(\mathrm{C} / \mathrm{EBP})$, beta (CEBPB) and MYC-associated factor X (MAX). The expression of these $11 \mathrm{TFs}$ in the three sample types is shown in Fig. 5. The results revealed that the expression of EGR1 was significantly higher in the hASCs than in the osteogenic and the myogenic lineages. Conversely, the expression of STAT3 was significantly lower in the hASCs than in the osteogenic and the myogenic lineages. Differential expression of the other 9 TFs among the three cell types was not found.

In addition, 26 and $21 \mathrm{TFs}$ were predicted to regulate the unshared up- and downregulated genes in the myogenic lineages, respectively. In the osteogenic lineages, 11 TFs were predicted to target the upregulated genes whereas only RAD21 was found to regulate the downregulated 


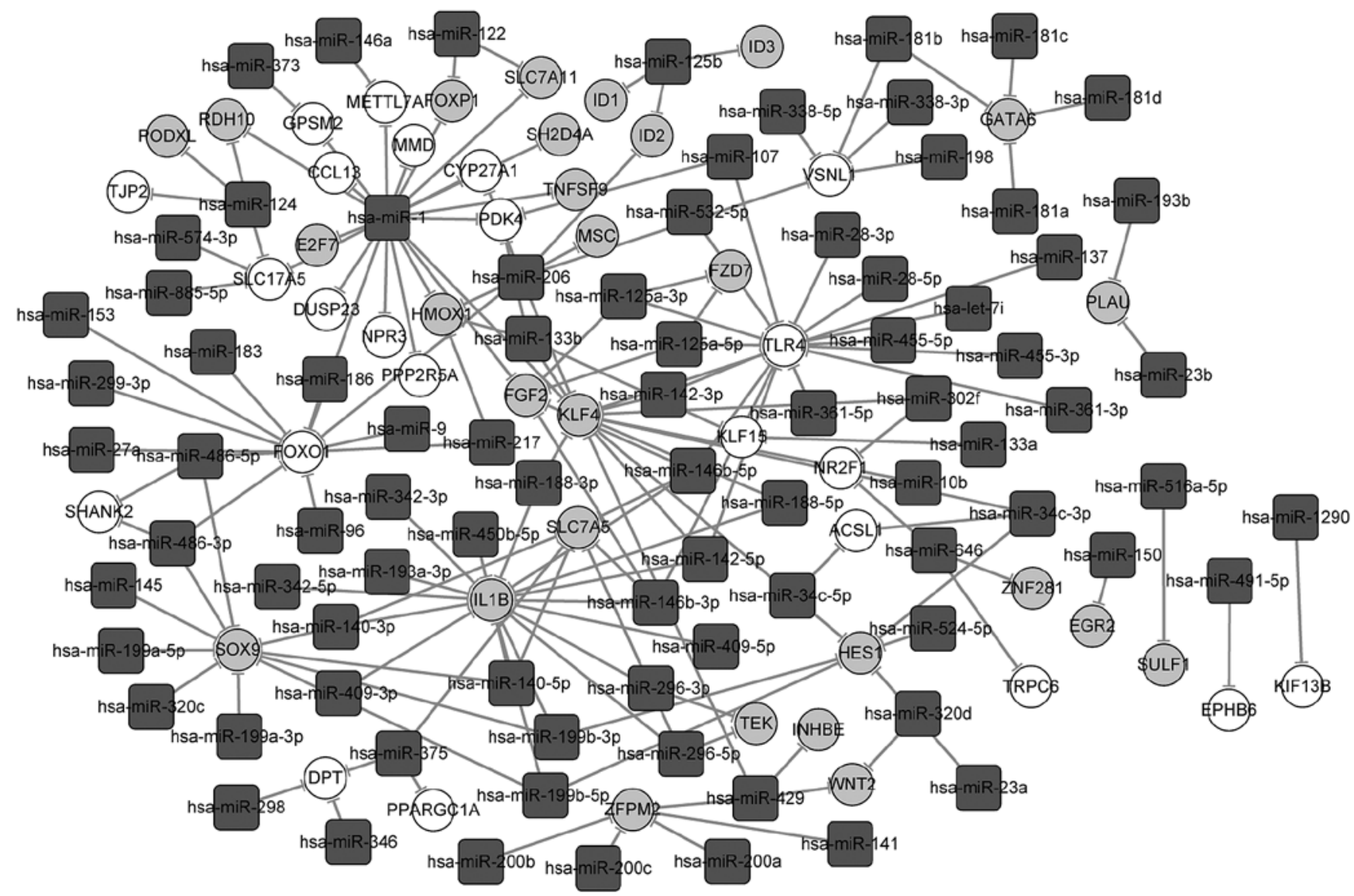

Figure 6. miRNA-target gene interaction network. The square nodes represent miRNA and the circle nodes represents the target gene. White nodes represent upregulated genes in myocytes and osteocytes. Light gray nodes represent downregulated genes in myocytes and osteocytes.

genes. Moreover, RAD21 was also included among the TFs regulating unshared downregulated genes in the myogenic lineages, including $V E G F A$ and SMAD family member 6 (SMAD6).

MiRNA-DEG interaction analysis. A total of 66 and 98 miRNA-mRNA pairs were finally screened out for the shared up- and downregulated genes in the osteogenic and the myogenic lineages to construct an miRNA-target gene interaction network, respectively (Fig. 6). In the network, hsa-miR-1, with the highest degree, regulated 20 common genes differentially expressed in the two cell types, including Forkhead box P1 (FOXP1), E2F transcription factor 7 (E2F7), chemokine (C-C motif) ligand 13 (CCL13), monocyte to macrophage differentiation-associated $(M M D)$ and pyruvate dehydrogenase kinase, isozyme 4 (PDK4). Moreover, the shared upregulated genes FOXO1, TLR 4 and downregulated gene $I L I B$ were regulated by $>9$ miRNAs during the differentiation of hASCs, and shared downregulated GATA6 was regulated by four hsa-miR-181 family members namely miR-181a, miR-181b, miR-181c and miR-181d.

Further, functional annotation revealed that the shared upregulated genes targeted by the predicted miRNAs were mainly involved in immune response-related BPs, including detection of fungus, and host defense responses. By contrast, the shared downregulated genes were significantly enriched in response to ozone, smooth muscle adaptation and regulation of myosin light chain kinase activity (Table VI).

\section{Discussion}

In the present study, we aimed to extend our understanding of the molecular mechanisms responsible for the differentiation of hASCs into myocytes and osteocytes. We found that four proteins encoded by $V E G F A, F G F 2, N G F$ and $I L I B$ were differentially expressed in the myogenic and the osteogenic lineages and presented in the PPI network at relatively high degrees. Moreover, the TF RAD21 was predicted to target both shared up- and downregulated genes as well as specific downregulated genes in the myogenic and the osteogenic lineages. In addition, miRNA-DEG interaction analysis revealed that hsa-miR-1 regulated the most shared DEGs in the two lineages, such as FOXPI and CCL13.

Previous findings have suggested that hASCs secrete significant numbers of angiogenic factors, including VEGFA (30). VEGFA is known to promote both angiogenesis and osteogenesis $(31,32)$. More recently, VEGFA has been proved to play an integral role in the crosstalk between endothelial cells and osteoblasts and is also considered as being of great importance for vascularization (33). VEGFA has been found to increase bone formation, promote osteoblast differentiation and inhibit the apoptosis of osteoblasts $(32,34)$. In addition, Song et al have identified VEGF as a critical factor in cardiomyogenesis in hASCs (35). FGF2, a member of the FGF family, has been identified as a major candidates for the regulation of self-renewal in human embryonic stem cells $(36,37)$. FGF2 may also be important in increasing the lifespan of bone marrow stromal cells and 
Table VI. Top 7 enriched GO terms in the BP category for target genes of miRNAs.

\begin{tabular}{|c|c|c|c|c|c|}
\hline & & GO ID & Name of BP & Count & P-value \\
\hline \multirow{7}{*}{$\begin{array}{l}\text { miRNA- } \\
\text { gene-up }\end{array}$} & \multirow[t]{7}{*}{$\mathrm{BP}$} & GO:0016046 & Detection of fungus & 16 & $3.05 \mathrm{E}-14$ \\
\hline & & GO:0052031 & Modulation by symbiont of host defense response & 16 & 8.69E-14 \\
\hline & & GO:0052033 & $\begin{array}{l}\text { Pathogen-associated molecular pattern dependent induction } \\
\text { by symbiont of host innate immune response }\end{array}$ & 16 & $8.69 \mathrm{E}-14$ \\
\hline & & GO:0052166 & Positive regulation by symbiont of host innate immune response & 16 & $8.69 \mathrm{E}-14$ \\
\hline & & GO:0052167 & Modulation by symbiont of host innate immune response & 16 & 8.69E-14 \\
\hline & & GO:0052169 & $\begin{array}{l}\text { Pathogen-associated molecular pattern dependent modulation } \\
\text { by symbiont of host innate immune response }\end{array}$ & 16 & $8.69 \mathrm{E}-14$ \\
\hline & & GO:0052255 & $\begin{array}{l}\text { Modulation by organism of defense response of other } \\
\text { organism involved in symbiotic interaction }\end{array}$ & 16 & $8.69 \mathrm{E}-14$ \\
\hline \multirow{7}{*}{$\begin{array}{l}\text { miRNA- } \\
\text { gene-down }\end{array}$} & \multirow[t]{7}{*}{$\mathrm{BP}$} & GO:0010193 & Response to ozone & 17 & 0 \\
\hline & & GO:0014805 & Smooth muscle adaptation & 21 & 0 \\
\hline & & GO:0035504 & Regulation of myosin light chain kinase activity & 17 & 0 \\
\hline & & GO:0035505 & Positive regulation of myosin light chain kinase activity & 17 & 0 \\
\hline & & GO:0060352 & Cell adhesion molecule production & 17 & 0 \\
\hline & & GO:0060353 & Regulation of cell adhesion molecule production & 17 & 0 \\
\hline & & GO:0060355 & Positive regulation of cell adhesion molecule production & 17 & 0 \\
\hline
\end{tabular}

Up, upregulated; down, downregulated; GO, Gene Ontology; BP, biological process;

for supporting proliferation as well as the chondrogenic and osteogenic differentiation potential $(38,39)$. Moreover, previous studies have shown that the exposure of hASCs to FGF2 led to the enhancement of chondrogenic lineage differentiation and the inhibition of osteogenic lineage differentiation, as well as the stimulation of adipogenic differentiation $(10,40,41)$. Notably, IL1B, which encodes an inflammatory cytokine, has been shown to be suppressed by mesenchymal stem cell (MSC) transplantation at the transcriptional and the post-transcriptional levels in myocardial infarction (42). NGF is also reported to be associated with many pathologic and physiologic processes, such as differentiation of stem cells (43). In this study, VEGFA, FGF2, IL1B and NGF were found to be downregulated in the myogenic and osteogenic lineages compared with hASCs and connected with relatively more DEGs in the PPI networks, which supports the hypothesis that there may be a correlation between these genes and the differentiation of hASCs.

Additionally, TFs and miRNAs are essential regulatory molecules after DNA replication involved in the differentiation of hASCs. The TF RAD21 has been proved to be associated with the maintenance of embryonic stem cell identity through association with the pluripotency transcriptional network (44). Consistent with our analysis, chromatin immunoprecipitation analysis was used in a previous study to confirm that VEGFA and SMAD6 expression is regulated by RAD21 (45). SMAD6, an inhibitory SMAD, has been reported to inhibit the TGF- $\beta$ signaling pathway that suppresses osteoblast and myogenic differentiation (46). The data from the present study revealed that RAD21 mediates the differentiation of hASCs by regulating the expression of VEGFA and SMAD6.

In a previous study, miR-1 was shown to strongly enhance myogenesis following the transfection of myoblasts with hsa-miR-1 by modulating skeletal muscle proliferation and differentiation (47). More importantly, hsa-miR-1 is required for smooth muscle cell lineage differentiation from embryonic stem cells by binding with the 3 ' untranslated region of the gene encoding Kruppel-like factor 4 (48). Following the construction of an miRNA-target gene interaction network, we found that miR-1 targeted FOXP1 in the differentiation of hASCs into osteocytes and myocytes, which is in agreement with the results of a previous study (49). Additionally, it was demonstrated that knockdown of FOXP1 suppressed the self-renewal capacity of MSCs and reduced the osteogenic potential (50). In the hASC-derived myocytes and osteocytes, CCL13 was upregulated which is consistent with the findings of a previous study revealing a 12-fold change after culturing hASCs with proinflammatory cytokines (51). Our results suggest that miR-1 modulates the differentiation of hASCs into myocytes and osteocytes by regulating FOXPI and CCL13.

In conclusion, we performed a comprehensive bioinformatics analysis of the expression profiles of in vitro-induced osteogenic and myogenic lineages and hASC cell lines from healthy donors. There may be a correlation between four shared downregulated genes in the two lineages, VEGFA, FGF2,ILIB and NGF, and the differentiation of hASCs. Notably, the TF RAD21 and hsa-miR-1 may play important roles in regulating the expression of differentiation-associated genes. This study may provide new insight into the underlying molecular mechanisms of hASC differentiation, which may help to repair and reconstruct damaged organs. However, further studies are warranted to confirm these results and to clarify their roles in the differentiation of hASCs.

\section{Acknowledgements}

The present study was supported by the Liaoning Province Science and Technology Research Project (no. 2013225220). 


\section{References}

1. Zuk PA, Zhu M, Mizuno H, Huang J, Futrell JW, Katz AJ, Benhaim P, Lorenz HP and Hedrick MH: Multilineage cells from human adipose tissue: implications for cell-based therapies. Tissue Eng 7: 211-228, 2001.

2. Halvorsen YD, Bond A, Sen A, Franklin DM, Lea-Currie YR, Sujkowski D, Ellis PN, Wilkison WO and Gimble JM: Thiazolidinediones and glucocorticoids synergistically induce differentiation of human adipose tissue stromal cells: biochemical, cellular, and molecular analysis. Metabolism 50: 407-413, 2001.

3. Zuk PA: The adipose-derived stem cell: looking back and looking ahead. Mol Biol Cell 21: 1783-1787, 2010.

4. Luzi E, Marini F, Sala SC, Tognarini I, Galli G and Brandi ML: Osteogenic differentiation of human adipose tissue-derived stem cells is modulated by the miR-26a targeting of the SMAD1 transcription factor. J Bone Miner Res 23: 287-295, 2008

5. Cai L, Johnstone BH, Cook TG, Tan J, Fishbein MC, Chen PS and March KL: IFATS collection: human adipose tissue-derived stem cells induce angiogenesis and nerve sprouting following myocardial infarction, in conjunction with potent preservation of cardiac function. Stem Cells 27: 230-237, 2009.

6. Nambu M, Ishihara M, Nakamura S, Mizuno H, Yanagibayashi S Kanatani Y, Hattori H, Takase B, Ishizuka T, Kishimoto S, et al: Enhanced healing of mitomycin C-treated wounds in rats using inbred adipose tissue-derived stromal cells within an atelocollagen matrix. Wound Repair Regen 15: 505-510, 2007.

7. Wilson A and Trumpp A: Bone-marrow haematopoietic-stem-cell niches. Nat Rev Immunol 6: 93-106, 2006.

8. Luu YK, Capilla E, Rosen CJ, Gilsanz V, Pessin JE, Judex S and Rubin CT: Mechanical stimulation of mesenchymal stem cell proliferation and differentiation promotes osteogenesis while preventing dietary-induced obesity. J Bone Miner Res 24: 50-61, 2009.

9. Lodish H, Flygare J and Chou S: From stem cell to erythroblast: regulation of red cell production at multiple levels by multiple hormones. IUBMB Life 62: 492-496, 2010.

10. Kakudo N, Shimotsuma A and Kusumoto K: Fibroblast growth factor-2 stimulates adipogenic differentiation of human adiposederived stem cells. Biochem Biophys Res Commun 359: 239-244, 2007.

11. Stewart AA, Byron CR, Pondenis H, Stewart MC: Effect of fibroblast growth factor-2 on equine mesenchymal stem cell monolayer expansion and chondrogenesis. Am J Vet Res 68 941-945, 2007.

12. Huang S, Wang S, Bian C, Yang Z, Zhou H, Zeng Y, Li H, Han Q and Zhao RC: Upregulation of miR-22 promotes osteogenic differentiation and inhibits adipogenic differentiation of human adipose tissue-derived mesenchymal stem cells by repressing HDAC6 protein expression. Stem Cells Dev 21: 2531-2540, 2012.

13. Mizuno H, Zuk PA, Zhu M, Lorenz HP, Benhaim P and Hedrick MH: Myogenic differentiation by human processed lipoaspirate cells. Plast Reconstr Surg 109: 199-111, 2002.

14. Planat-Bénard V, Menard C, André M, Puceat M, Perez A, Garcia-Verdugo JM, Pénicaud L and Casteilla L: Spontaneous cardiomyocyte differentiation from adipose tissue stroma cells. Circ Res 94: 223-229, 2004.

15. Jeon ES, Moon HJ, Lee MJ, Song HY, Kim YM, Bae YC, Jung JS and Kim JH: Sphingosylphosphorylcholine induces differentiation of human mesenchymal stem cells into smooth-muscle-like cells through a TGF-beta-dependent mechanism. J Cell Sci 119: 4994-5005, 2006.

16. Lee WC, Rubin JP and Marra KG: Regulation of alpha-smooth muscle actin protein expression in adipose-derived stem cells Cells Tissues Organs 183: 80-86, 2006.

17. Kim YJ, Bae SW, Yu SS, Bae YC and Jung JS: miR-196a regulates proliferation and osteogenic differentiation in mesenchymal stem cells derived from human adipose tissue. J Bone Miner Res 24 816-825, 2009

18. Maroni P, Brini AT, Arrigoni E, de Girolamo L, Niada S, Matteucci E, Bendinelli P and Desiderio MA: Chemical and genetic blockade of HDACs enhances osteogenic differentiation of human adipose tissue-derived stem cells by oppositely affecting osteogenic and adipogenic transcription factors Biochem Biophys Res Commun 428: 271-277, 2012.

19. Berdasco M, Melguizo C, Prados J, Gómez A, Alaminos M, Pujana MA, Lopez M, Setien F, Ortiz R, Zafra I, et al: DNA methylation plasticity of human adipose-derived stem cells in lineage commitment. Am J Pathol 181: 2079-2093, 2012.
20. Irizarry RA, Hobbs B, Collin F, Beazer-Barclay YD, Antonellis KJ, Scherf U and Speed TP: Exploration, normalization, and summaries of high density oligonucleotide array probe level data. Biostatistics 4: 249-264, 2003.

21. Smyth GK: Limma: linear models for microarray data. In: Bioinformatics and Computational Biology Solutions Using $\mathrm{R}$ and Bioconductor. Springer, New York, pp397-420, 2005.

22. Ashburner M, Ball CA, Blake JA, Botstein D, Butler $\mathrm{H}$, Cherry JM, Davis AP, Dolinski K, Dwight SS, Eppig JT, et al: Gene ontology: Tool for the unification of biology. The Gene Ontology Consortium. Nat Genet 25: 25-29, 2000.

23. Kanehisa M and Goto S: KEGG: Kyoto encyclopedia of genes and genomes. Nucleic Acids Res 28: 27-30, 2000.

24. Wang J, Zhou X, Zhu J, Gu Y, Zhao W, Zou J and Guo Z: GO-function: deriving biologically relevant functions from statistically significant functions. Brief Bioinform 13: 216-227, 2012.

25. Franceschini A, Szklarczyk D, Frankild S, Kuhn M, Simonovic M, Roth A, Lin J, Minguez P, Bork P, von Mering C and Jensen LJ: STRING v9.1: protein-protein interaction networks, with increased coverage and integration. Nucleic Acids Res 41: D808-D815, 2013.

26. Kohl M, Wiese S and Warscheid B: Cytoscape: software for visualization and analysis of biological networks. Methods Mol Biol 696: 296-303, 2011

27. Meyer LR, Zweig AS, Hinrichs AS, Karolchik D, Kuhn RM, Wong M, Sloan CA, Rosenbloom KR, Roe G, Rhead B, et al: The UCSC Genome Browser database: extensions and updates 2013. Nucleic Acids Res 41: D64-D69, 2013

28. Xiao F, Zuo Z, Cai G, Kang S, Gao X and Li T: miRecords: an integrated resource for microRNA-target interactions. Nucleic Acids Res 37: D105-D110, 2009.

29. Dweep H, Sticht C, Pandey P and Gretz N: miRWalk - database: prediction of possible miRNA binding sites by 'walking' the genes of three genomes. J Biomed Inform 44: 839-847, 2011.

30. Rehman J, Traktuev D, Li J, Merfeld-Clauss S, Temm-Grove CJ, Bovenkerk JE, Pell CL, Johnstone BH, Considine RV and March KL: Secretion of angiogenic and antiapoptotic factors by human adipose stromal cells. Circulation 109: 1292-1298, 2004.

31. Olsson AK, Dimberg A, Kreuger J and Claesson-Welsh L: VEGF receptor signalling - in control of vascular function. Nat Rev Mol Cell Biol 7: 359-371, 2006.

32. Street J, Bao M, deGuzman L, Bunting S, Peale FV Jr, Ferrara N, Steinmetz H, Hoeffel J, Cleland JL, Daugherty A, et al: Vascular endothelial growth factor stimulates bone repair by promoting angiogenesis and bone turnover. Proc Natl Acad Sci USA 99: 9656-9661, 2002

33. Clarkin CE, Emery RJ, Pitsillides AA and Wheeler-Jones CP Evaluation of VEGF-mediated signaling in primary human cells reveals a paracrine action for VEGF in osteoblast-mediated crosstalk to endothelial cells. J Cell Physiol 214: 537-544, 2008.

34. Street J and Lenehan B: Vascular endothelial growth factor regulates osteoblast survival - evidence for an autocrine feedback mechanism. J Orthop Surg 4: 19, 2009.

35. Song YH, Gehmert S, Sadat S, Pinkernell K, Bai X, Matthias N and Alt E: VEGF is critical for spontaneous differentiation of stem cells into cardiomyocytes. Biochem Biophys Res Commun 354: 999-1003, 2007.

36. Xu C, Rosler E, Jiang J, Lebkowski JS, Gold JD, O'Sullivan C, Delavan-Boorsma K, Mok M, Bronstein A and Carpenter MK: Basic fibroblast growth factor supports undifferentiated human embryonic stem cell growth without conditioned medium. Stem Cells 23: 315-323, 2005

37. Dvorak P, Dvorakova D, Koskova S, Vodinska M, Najvirtova M, Krekac D and Hampl A: Expression and potential role of fibroblast growth factor 2 and its receptors in human embryonic stem cells. Stem Cells 23: 1200-1211, 2005.

38. Martin I, Muraglia A, Campanile G, Cancedda R and Quarto R: Fibroblast growth factor-2 supports ex vivo expansion and maintenance of osteogenic precursors from human bone marrow. Endocrinology 138: 4456-4462, 1997.

39. Solchaga LA, Penick K, Porter JD, Goldberg VM, Caplan AI and Welter JF: FGF-2 enhances the mitotic and chondrogenic potentials of human adult bone marrow-derived mesenchymal stem cells. J Cell Physiol 203: 398-409, 2005.

40. Quarto N and Longaker MT: FGF-2 inhibits osteogenesis in mouse adipose tissue-derived stromal cells and sustains their proliferative and osteogenic potential state. Tissue Eng 12: $1405-1418,2006$ 
41. Chiou M, Xu Y and Longaker MT: Mitogenic and chondrogenic effects of fibroblast growth factor-2 in adipose-derived mesenchymal cells. Biochem Biophys Res Commun 343: 644-652, 2006.

42. Guo J, Lin GS, Bao CY, Hu ZM and Hu MY: Anti-inflammation role for mesenchymal stem cells transplantation in myocardial infarction. Inflammation 30: 97-104, 2007.

43. Sariola H: The neurotrophic factors in non-neuronal tissues. Cell Mol Life Sci 58: 1061-1066, 2001.

44. Nitzsche A, Paszkowski-Rogacz M, Matarese F, Janssen-Megens EM, Hubner NC, Schulz H, de Vries I, Ding L, Huebner N, Mann M, et al: RAD21 cooperates with pluripotency transcription factors in the maintenance of embryonic stem cell identity. PLoS One 6: e19470, 2011.

45. Tang M, Chen B, Lin T, Li Z, Pardo C, Pampo C, Chen J, Lien CL, Wu L, Ai L, et al: Restraint of angiogenesis by zinc finger transcription factor CTCF-dependent chromatin insulation. Proc Natl Acad Sci USA 108: 15231-15236, 2011.

46. Roelen BA and Dijke P: Controlling mesenchymal stem cell differentiation by TGFBeta family members. J Orthop Sci 8: 740-748, 2003.

47. Chen JF, Mandel EM, Thomson JM, Wu Q, Callis TE, Hammond SM, Conlon FL and Wang DZ: The role of microRNA-1 and microRNA-133 in skeletal muscle proliferation and differentiation. Nat Genet 38: 228-233, 2006.
48. Xie C, Huang H, Sun X, Guo Y, Hamblin M, Ritchie RP, Garcia-Barrio MT, Zhang J and Chen YE: MicroRNA-1 regulates smooth muscle cell differentiation by repressing Kruppel-like factor 4. Stem Cells Dev 20: 205-210, 2011.

49. Datta J, Kutay H, Nasser MW, Nuovo GJ, Wang B, Majumder S, Liu CG, Volinia S, Croce CM, Schmittgen TD, et al: Methylation mediated silencing of MicroRNA-1 gene and its role in hepatocellular carcinogenesis. Cancer Res 68: 5049-5058, 2008.

50. Kubo H, Shimizu M, Taya Y, Kawamoto T, Michida M, Kaneko E, Igarashi A, Nishimura M, Segoshi K, Shimazu Y, et al: Identification of mesenchymal stem cell (MSC)-transcription factors by microarray and knockdown analyses, and signature molecule-marked MSC in bone marrow by immunohistochemistry. Genes Cells 14: 407-424, 2009.

51. Crop MJ, Baan CC, Korevaar SS, Ijzermans JN, Pescatori M, Stubbs AP, van Ijcken WF, Dahlke MH, Eggenhofer E, Weimar W and Hoogduijn MJ: Inflammatory conditions affect gene expression and function of human adipose tissue-derived mesenchymal stem cells. Clin Exp Immunol 162: 474-486, 2010. 